\title{
Development of a railway signaling device based on mixed digital and analog signals using digital signal processors
}

\author{
R. Ishikawa ${ }^{1}$, D. Koshino ${ }^{2}$, H. Mochizuki ${ }^{2}$, S. Takahashi ${ }^{2}$, \\ H. Nakamura ${ }^{2}$, S. Nishida ${ }^{1} \&$ M. Sano ${ }^{1}$ \\ ${ }^{1}$ Kyosan Electric Mfg. Co., Ltd., Japan \\ ${ }^{2}$ College of Science and Technology, Nihon University, Japan
}

\begin{abstract}
In Japan, automatic train control (ATC) systems, one type of railway signaling system, transmit train control information by using analog signals based on amplitude modulation (AM) in the audio frequency band. To realize highly functional train control by increasing the data transmission speed, there have been many studies on digital ATC that transmits train control information by using digital signals based on phase shift keying (PSK), and these systems are employed in some railway lines. In practice, however, it is difficult to install digital ATC because it is impossible to ensure another transmission band for digital ATC signals due to the existing track circuit configuration and interoperability conditions, and there are many railway lines in which analog ATC is still employed. To overcome this restriction, we proposed a novel railway signaling system using mixed digital and analog signals. We employed quadrature PSK (QPSK) for digital signals and developed a transmission device using digital signal processors. We evaluated the transmission characteristics by conducting a basic experiment.

Keywords: railway signaling, amplitude modulation, phase shift keying, digital signal processor.
\end{abstract}

\section{Introduction}

In Japan, automatic train control (ATC) systems ensure railway safety by transmitting train control data via the rails based on amplitude modulation (AM) in the audio frequency band. There is currently a great deal of research on digital ATC, 
which transmits train control information based on digital modulation schemes, such as phase shift keying (PSK) [1]. Since digital ATC can realize highly functional train control by increasing the data transmission speed, many railway engineers are attempting to install digital ATC. In our previous work, we proposed a high-speed data transmission system employing code-division multiple access (CDMA) and quadrature amplitude modulation (QAM) for digital ATC [2].

In practice, however, there are some problems in installing digital ATC. In railway signaling via rails, the audio frequency band is usually employed for data transmission, and the carrier frequency depends on the track circuit configuration and interoperability conditions. Therefore, since it is difficult to ensure a new channel for digital ATC signals in a railway line in which analog ATC is employed, all equipment must be replaced simultaneously when attempting to install digital ATC.

To overcome this restriction, we investigated a novel data transmission scheme for railway signaling. Since a digital modulation signal such as PSK has no amplitude component, we considered that conventional analog ATC can employ it as an AM carrier. Based on this idea, we previously proposed a data transmission scheme based on mixed digital and analog signals, which we call "digital-analog ATC".

In the present study, we attempted to develop a transceiver for digital-analog ATC using digital signal processors (DSPs). In the transmitter, we implemented an AM modulator that uses a quadrature PSK (QPSK) signal as an AM carrier. In the receiver, on the other hand, we implemented some functions such as automatic gain control (AGC) and a Costas loop, which is one carrier synchronization method, for QPSK demodulation. In addition, we conducted a basic experiment to verify these transceiver functions. We also conducted an experiment using a setup including conventional equipment and evaluated the spectral distribution of the AM demodulated signal and the QPSK constellation characteristics.

\section{Overview of digital-analog ATC}

\subsection{Definition of digital-analog signal}

Figure 1 shows the scheme for generating a so-called digital-analog signal. First, it is necessary to be able to receive the train control signal with conventional ATC equipment. Therefore, the scheme shown in Figure 1 is based on an AM transmitter. It is possible to demodulate an AM signal even if a PSK signal that has no amplitude component is employed as the AM carrier. Based on this idea, we developed the digital-analog signal as a novel signal generation scheme that applies a PSK -based modulation signal to an AM-carrier of an AM-based transceiver.

\subsection{Composition of digital-analog ATC}

Figure 2 shows a block diagram of the digital-analog ATC. In this figure, a digitalanalog signal generator installed as field equipment is connected with the rail 


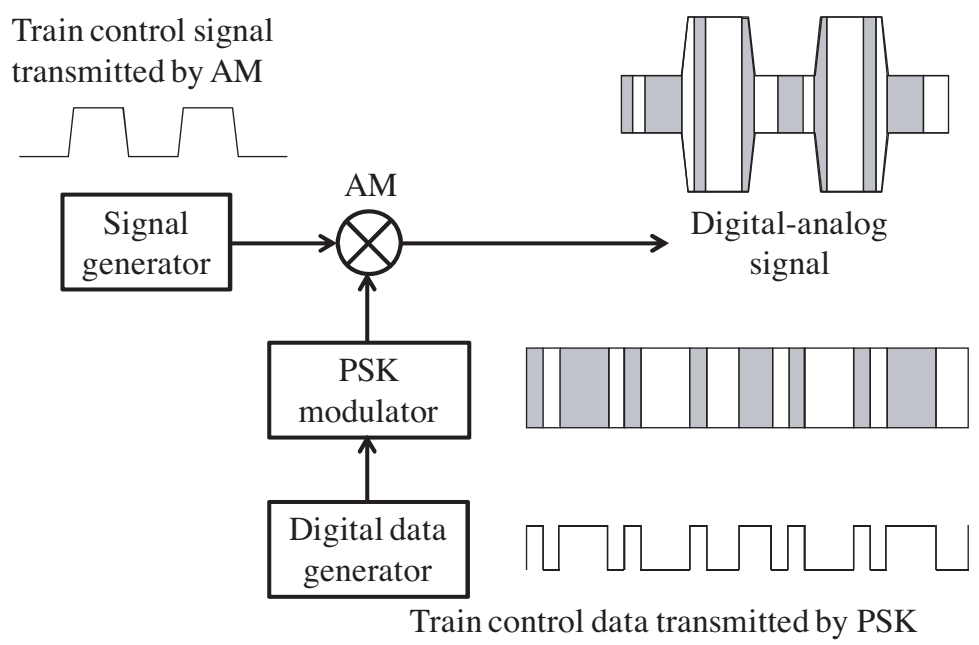

Figure 1: Generation of digital-analog signal.

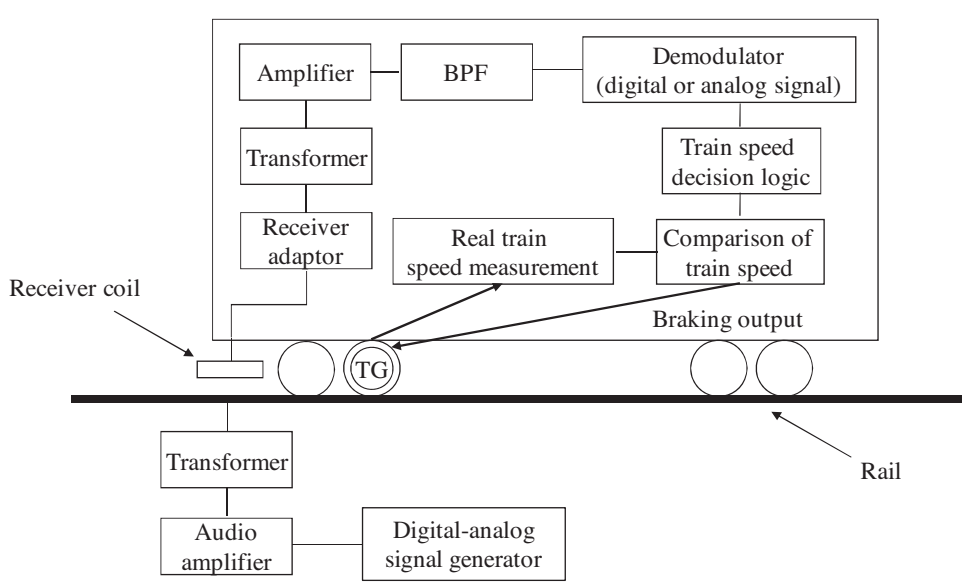

Figure 2: Block diagram of digital-analog ATC.

serving as the transmission medium. On the train, the received signal is demodulated after passing through an amplifier and a band pass filter (BPF). Since the received signal includes digital and analog signals, either a digital signal demodulator or an analog signal demodulator is installed. The instructed train speed is determined from the demodulated signal and is compared with the actual train speed measured by a tachogenerator (TG). If the actual train speed is faster than this instructed train speed, it is reduced by applying the brake. 


\subsection{Characteristics of digital-analog ATC}

When installing digital ATC, one problem is that all equipment must be replaced simultaneously. With our proposed digital-analog ATC, on the other hand, it is possible to transmit mixed digital and analog signals using the same carrier frequency. Therefore, this scheme has the following benefits:

- It is possible to replace existing equipment with digital ATC equipment gradually.

- It is easy to conduct verification experiments for installing digital ATC.

- It is possible to verify the validity of digital ATC signals by comparing them with analog ATC signals.

However, the AM power spectrum is distributed to other frequency bands by employing a digitally modulated signal as the carrier. Therefore, digital-analog ATC requires higher signal power to ensure an adequate signal-to-noise ratio $(\mathrm{S} / \mathrm{N})$.

\section{Design of transmission device using digital signal processors}

\subsection{Overview of transmission device development}

We attempted to develop a transmission device based on the ideas described in the previous section. Since the transmission band is in the audio frequency band, we used digital signal processors (DSPs), which have many applications in audio signal processing. Since one goal of our research is to replace analog ATC with digital ATC smoothly, in our experiments we used a conventional analog ATC receiver, and we developed a digital-analog transmitter and a digital ATC receiver.

\subsection{Design of digital-analog transmitter}

As mentioned above, since a PSK-based signal is employed as the AM carrier for the digital-analog signal, we designed the digital-analog transmitter to include both AM and PSK modulators. In addition, we adopted quadrature PSK (QPSK) to increase the digital data transmission speed. In QPSK, two orthogonal carrier signals are used to transmit digital data. One is given by $\cos 2 \pi f_{c} t$, and the other is given by $\sin 2 \pi f_{c} t$. The two carrier signals remain orthogonal in one period:

$$
\int_{0}^{T_{c}} \cos 2 \pi f_{c} t \times \sin 2 \pi f_{c} t d t=0
$$

where $T_{c}$ is the period of the carrier signals, which is equal to $1 / f_{c}$. By using $\cos 2 \pi f_{c} t$ and $\sin 2 \pi f_{c} t$, the QPSK signal is given by:

$$
s(t)=\frac{1}{\sqrt{2}} d_{I}(t) \cos \left(2 \pi f_{c} t\right)+\frac{1}{\sqrt{2}} d_{Q}(t) \sin \left(2 \pi f_{c} t\right) .
$$

The channel in which $\cos 2 \pi f_{c} t$ is used as a carrier signal is generally called the inphase channel, or I channel, and the channel in which $\sin 2 \pi f_{c} t$ is used as a carrier 


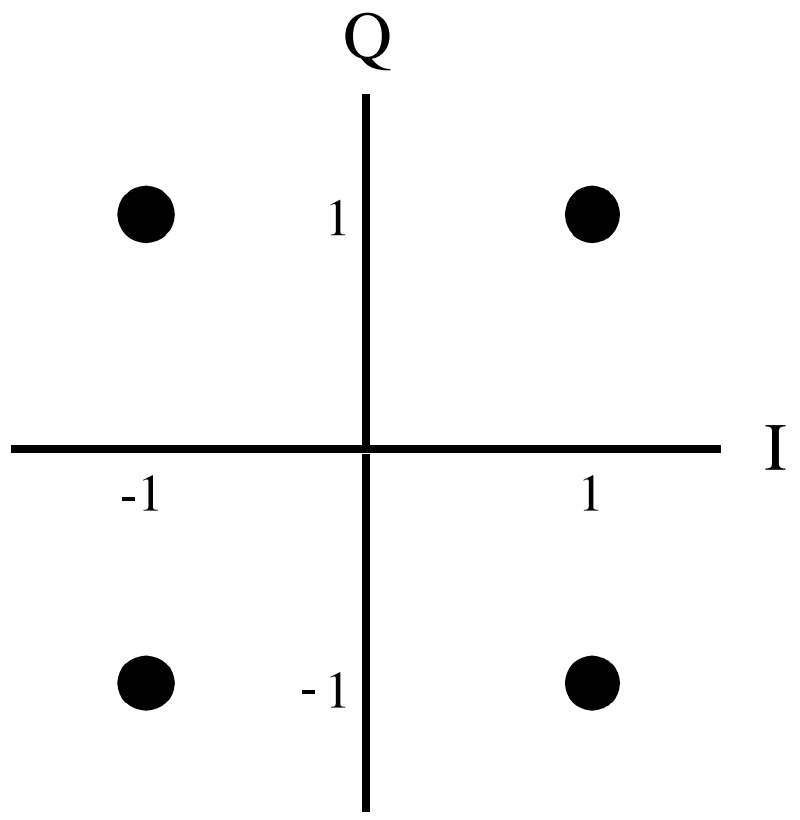

Figure 3: Symbol placement in QPSK.

signal is generally called the quadrature-phase channel, or Q channel. The data in the I and Q channels are $d_{I}(t)$ and $d_{Q}(t)$, respectively[3]. Figure 3 shows the symbol placement in QPSK. In this figure, QPSK transmits two bits simultaneously by assigning one bit, 1 or -1 , to the I channel and Q channel, respectively.

\subsection{Design of a digital ATC receiver}

As mentioned above, since the digital-analog signal includes an AM signal, we need to implement a function to cancel an amplitude component in a digital ATC receiver. To normalize the amplitude component, we applied automatic gain control (AGC) in a digital ATC receiver developed using a DSP. Specifically, we developed software to implement a function for squared detection, which is an AM detection method.

We used a Costas loop as the carrier synchronization method and implemented it in the digital ATC receiver. A Costas loop is based on a phase locked loop (PLL), as shown in Figure 4. Since QPSK has four symbols at intervals of $\pi / 2$, as shown in Figure 3, the symbol element is cancelled by multiplying the phase by four at the Costas loop. The Costas loop detects an output signal that is proportional to the phase difference between the received signal and a voltage controlled oscillator (VCO) signal and ensures carrier synchronization by adjusting the phase difference to zero. 


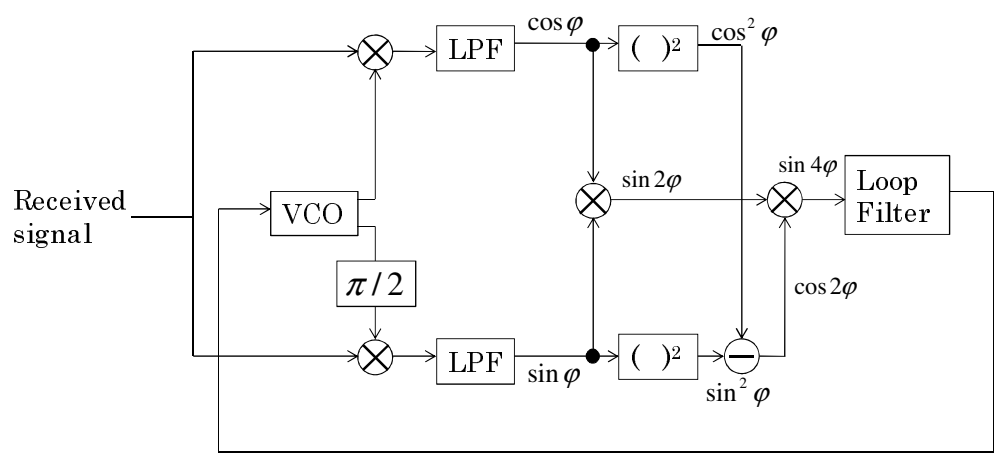

Figure 4: Block diagram of Costas loop.

\section{Evaluation of digital-analog ATC transceiver}

\subsection{Specifications}

The specifications of a digital-analog ATC transceiver developed based on the design in the previous section are shown in Table 1. Although we set the carrier frequency to $3,150 \mathrm{~Hz}$ in this study, other frequencies may be used in practice, for example, $5,250 \mathrm{~Hz}$. Similarly, we used $35 \mathrm{~Hz}$ as the analog signal frequency, but other frequencies may be used in practice, such as $28 \mathrm{~Hz}, 64 \mathrm{~Hz}$, etc. The digitalanalog ATC transceiver that we developed can freely set these values, as well as the digital transmission speed, by changing the DSP parameters.

\subsection{Verification of basic functions in digital-analog ATC transceiver}

In order to verify the basic functions, such as AGC and carrier synchronization, we conducted a basic experiment in which a transmitter was connected directly to a receiver based on the specifications shown in Table 1. Figure 5 shows the

Table 1: Specifications of digital-analog ATC transceiver.

\begin{tabular}{|l|c|}
\hline Parameter & Value \\
\hline Carrier frequency & $3,150 \mathrm{~Hz}$ \\
\hline Analog modulation method & $\mathrm{AM}$ \\
\hline Digital modulation method & QPSK \\
\hline Analog signal frequency & $35 \mathrm{~Hz}$ \\
\hline Digital transmission speed & $400 \mathrm{bps}$ \\
\hline Sampling frequency of DSPs & $48 \mathrm{kHz}$ \\
\hline
\end{tabular}




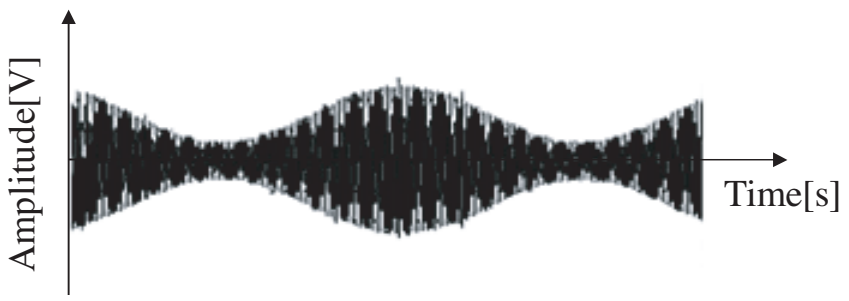

(a) Received signal.

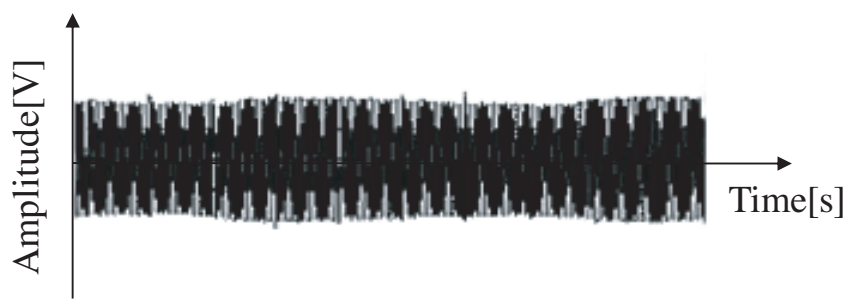

(b) Received signal after applying AGC function.

Figure 5: Waveforms at the digital ATC receiver.

waveforms at the digital ATC receiver. In this figure, we verified that the amplitude component of the digital-analog signal was cancelled by applying the AGC function. Figure 6 shows the constellation characteristics after QPSK demodulation. The effectiveness of the AGC function was also verified from this figure because the amplitude of the demodulated signal was approximately constant. In addition, the phase of the demodulated signal was also approximately constant, showing that the VCO output at the receiver could be synchronized with the carrier of the received signal. We verified that the function of the Costas loop could be implemented in software on the DSP.

\subsection{Experiment using actual railway signaling devices}

To verify the characteristics of a conventional analog ATC receiver when presented with the digital-analog signal, we conducted an experiment using actual railway signaling devices, as shown in Figure 7. After the digital-analog signal generated by the digital-analog ATC transmitter passed through a bandpass filter (BPF), which is typically employed as the receiver unit on actual trains, it was split and supplied to a conventional analog ATC receiver and the digital ATC receiver that we developed. Figure 8 shows the signal after passing through the BPF, which had narrow band characteristics, and Figure 9 shows the spectral distribution for the AM demodulated signal. In these figures, since the QPSK signal was influenced by the BPF characteristics, the power spectrum increases to cover a wide bandwidth, not just $35 \mathrm{~Hz}$, which is the analog signal frequency shown in Table 1. 


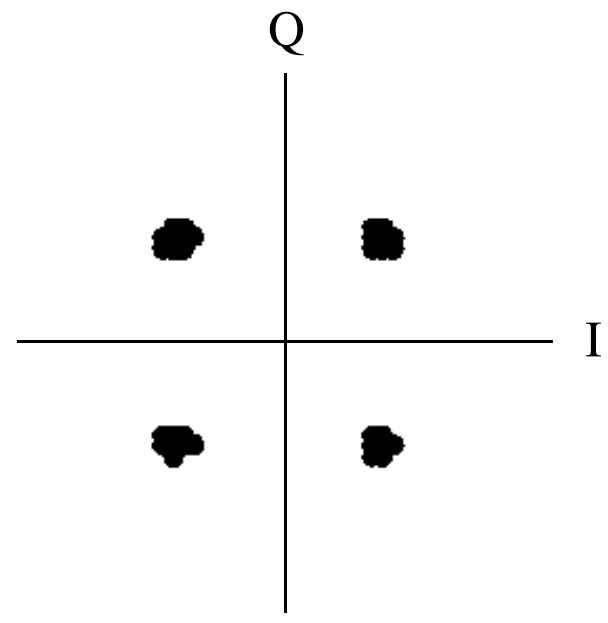

Figure 6: Constellation characteristics after QPSK demodulation.

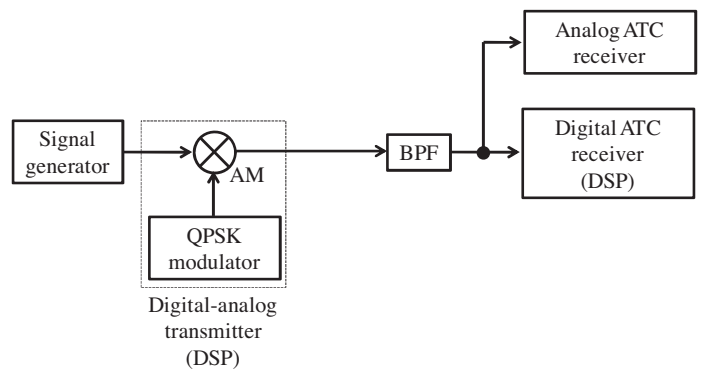

Figure 7: Experimental setup, including actual railway signaling devices.

However, since the power at $35 \mathrm{~Hz}$ was much larger than that ather frequencies, it was adequate for detecting the train control signal. We verified that the correct signal corresponding to $35 \mathrm{~Hz}$ could be detected with the setup shown in Figure 7.

\section{Future deployment of highly functional ATC system}

As mentioned above, adoption of a digital-analog signal can realize a highly functional ATC system that is free of restrictions due to the track circuit configuration and interoperability conditions. We noted that the PSK signal has no amplitude component, and we employed it in analog ATC using AM. Therefore, once 


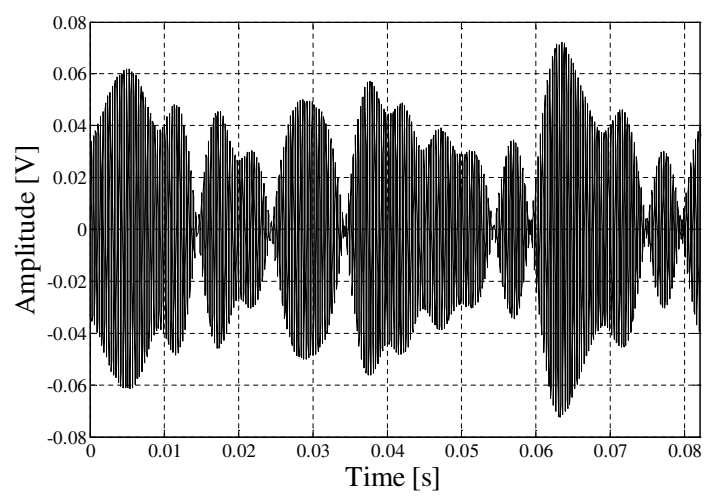

Figure 8: Mixed-signal waveform, including AM signal and digital signal.

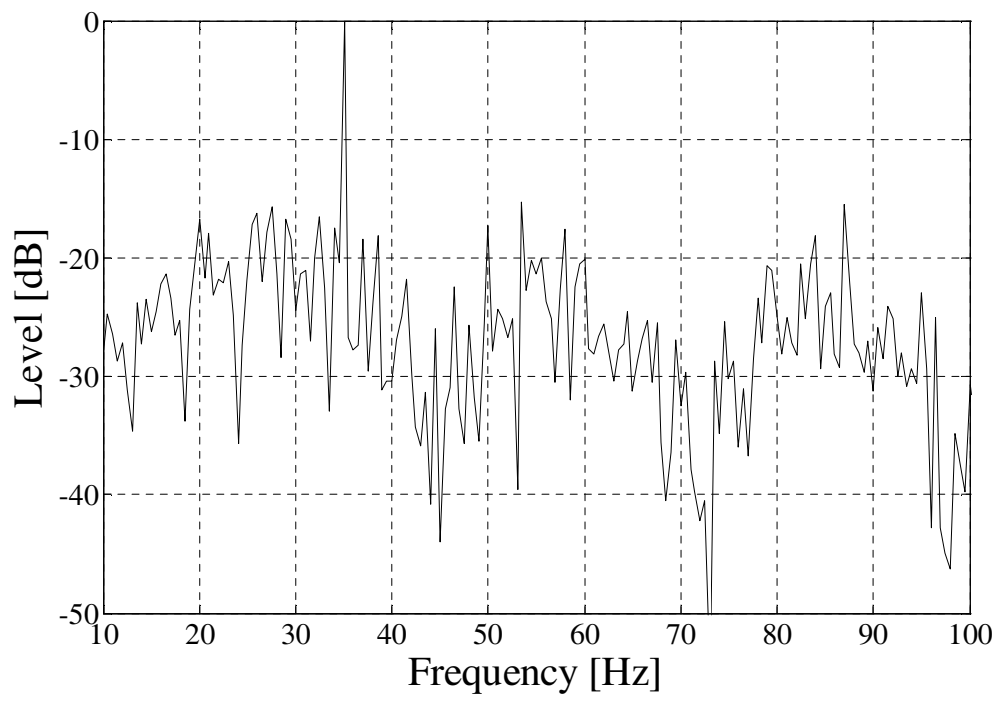

Figure 9: Spectral distribution of AM demodulation.

existing equipment is completely replaced with digital ATC, we will be able to use the amplitude component for a digital ATC system in order to increase the transmission speed. At present, we are developing a transceiver using QAM for a digital ATC system. Since QAM can realize high-capacity data transmission compared with PSK by making use of the amplitude component, digital-analog ATC has the potential to realize more highly functional systems by updating the transceiver that we developed. 


\section{Conclusion}

In this study, we proposed a transmission system that mixes digital and analog signals in the same frequency band, called "digital-analog ATC", as a highly functional ATC system that is free of the restrictions caused by the track circuit configuration and interoperability conditions.

We designed a digital-analog ATC transceiver including some functions, such as AGC and a Costas loop, developed using DSPs. From the result of a simple experiment, we verified the basic functions of the digital-analog ATC transceiver. In addition, in a setup including actual railway signaling devices, when a digitalanalog signal was given to a conventional analog ATC receiver, the correct signal corresponding to the AM signal frequency could be detected.

In future research, we plan to evaluate the proposed system quantitatively by studying the $\mathrm{S} / \mathrm{N}$ ratio characteristics. We will also investigate a detailed procedure for implementing an actual ATC system.

\section{References}

[1] S. Irie and T. Hasegawa: "A study on the Railway Signalling System using Spread Spectrum Communication", IEICE Technical Report, Vol. 93, No. 89, pp. 43-48 (1993).

[2] H. Mochizuki, S. Takahashi, H. Nakamura, S. Nishida and R. Ishikawa: "Development of a High-speed Rail Transmission System Using Digital Signal Processors for Railway Signalling", Eleventh International Conference on Computer System Design and Operation in the Railway and Other Transit Systems, pp. 295-304 (2008).

[3] H. Harada and R. Prasad: Simulation and Software Radio for Mobile Communications, Artech House, pp. 90-91 (2002). 